\title{
Simply mixing poly-protein $G$ with detection antibodies enhances the detection limit and sensitivity of immunoassays
}

Yi-Jou Chen ${ }^{\dagger}$, Michael Chen ${ }^{\dagger}$, Tian-Lu Cheng ${ }^{\dagger}, \perp$, Steve R. Roffler ${ }^{\circ}$, Shyr-Yi Lin ${ }^{\epsilon, \bar{T}, ~}$, , Hui-Lan Hsu ${ }^{\S}$, Chang-Hung Wang ${ }^{\dagger}$, Che-Yi Chen ${ }^{\dagger}$, An-Pei Kao ${ }^{2}$ Jing-Jy Cheng ${ }^{\dagger, \infty}$, Kuo-Hsiang Chuang ${ }^{\dagger}, \oint_{,}, \Delta,{ }^{*}$

$\dagger$ Ph.D. Program in Clinical Drug Development of Herbal Medicine, Taipei Medical University, Taipei, Taiwan

$\$$ Center for Biomarkers and Biotech Drugs, Kaohsiung Medical University, Kaohsiung, Taiwan.

${ }^{\perp}$ Department of Biomedical Science and Environmental Biology, Kaohsiung Medical University, Kaohsiung, Taiwan.

${ }^{\circ}$ Institute of Biomedical Sciences, Academia Sinica, Taipei, Taiwan

${ }^{€}$ Department of Primary Care Medicine, Taipei Medical University Hospital, Taipei, Taiwan

${ }^{\bar{T}}$ Department of General Medicine, School of Medicine, College of Medicine, Taipei Medical University, Taipei, Taiwan,

${ }^{z}$ TMU Research Center of Cancer Translational Medicine, Taipei Medical University, Taipei, Taiwan, ROC;

$\oint$ Graduate Institute of Pharmacognosy, Taipei Medical University, Taipei, Taiwan

${ }^{7}$ Stemforce Biotechnology Co., Ltd, Chiayi City, Taiwan

${ }^{\infty}$ National Research Institute of Chinese Medicine, Ministry of Health and Welfare, Taipei, Taiwan.

\# Ph.D. Program in Biotechnology Research and Development, Taipei Medical University, Taipei, Taiwan

${ }^{\Delta}$ Traditional Herbal Medicine Research Center of Taipei Medical University Hospital, Taipei Medical University, Taipei, Taiwan

* Corresponding Author

Dr. Kuo-Hsiang Chuang, Graduate Institute of Pharmacognosy, Taipei Medical University, 250 Wu-Hsing Street, Taipei, TAIWAN

Phone: 886-2-27361663-6163, E-mail: khchuang@,tmu.edu.tw 


\section{Table of Contents}

\section{Experimental section}

The interference of bovine serum albumin (BSA) and bovine calf serum (BCS) blocking solutions on 8pG based ELISA.

Figure S1. The interference of blocking reagents for the sensitivity and background value of 8pG based ELISAs

Figure S2. Models of antibody/8pG complexes "standing upright" or "lying down" on (A) high density or (B) low density antigens.

Figure S3. The raw images of Western blot data in the presence and absence of $8 \mathrm{pG}$. 


\section{Experimental section}

The interference of bovine serum albumin (BSA) and bovine calf serum (BCS) blocking solutions on 8pG based ELISA. The $\mathrm{PEG}_{5 \mathrm{~K}}-\mathrm{NH}_{2}$ serially diluted $(0.69,2,6.2,18.5,55.5,166$ and $500 \mathrm{ng} / \mathrm{well})$ in coating buffer $\left(0.1 \mathrm{M} \mathrm{NaHCO}_{3}, \mathrm{pH}=9\right)$ was coated in Maxisorp 96-well microplates for 2 hours at $37^{\circ} \mathrm{C}$. The microplates were blocked with $3 \%(\mathrm{w} / \mathrm{v}) \mathrm{BSA}$ or $3 \%(\mathrm{w} / \mathrm{v}) \mathrm{BCS}$ in PBS overnight at $4{ }^{\circ} \mathrm{C}$. Biotinconjugated anti-PEG antibodies ( $2 \mu \mathrm{g} / \mathrm{mL}$ of 3.3-biotin) were mixed individually by a 1:1 volume ratio with $8 \mathrm{pG}$ at room temperature for 2 hours (the final concentrations of 3.3-biotin was $1 \mu \mathrm{g} / \mathrm{mL}$ ). The $8 \mathrm{pG} / 3.3$ biotin mixtures were added to microplates and incubated at room temperature for 1 hour. After washing the microplates, streptavidin-HRP and ABTS were sequentially added and incubated in the microplates to detect $\mathrm{PEG}_{5 \mathrm{~K}}-\mathrm{NH}_{2}$. Color development was measured at $405 \mathrm{~nm}$ by a microplate reader.

A

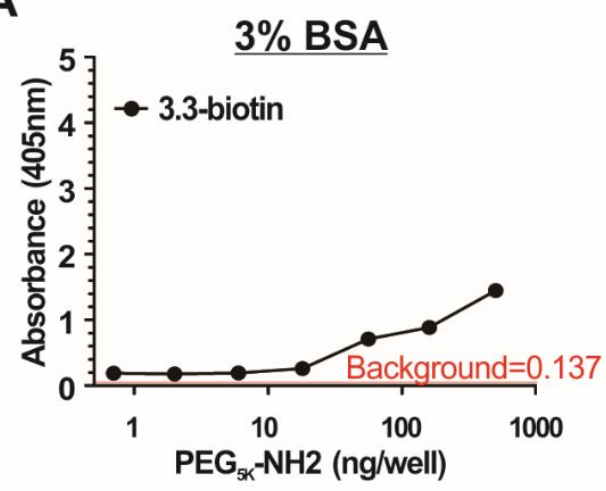

C

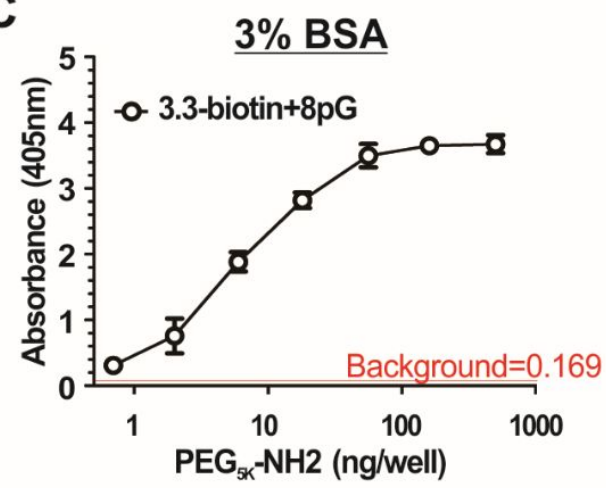

B

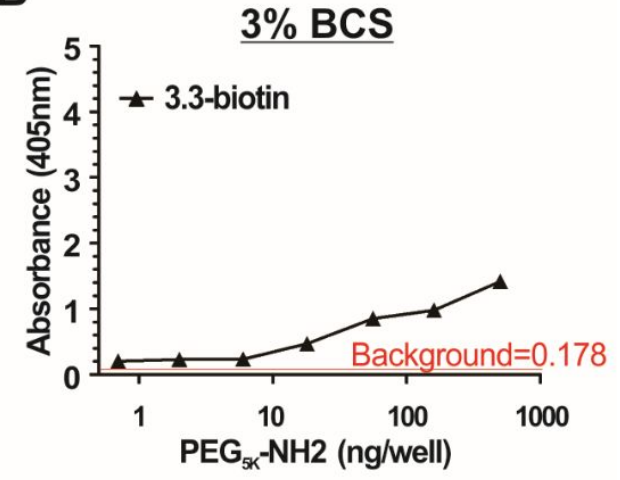

D

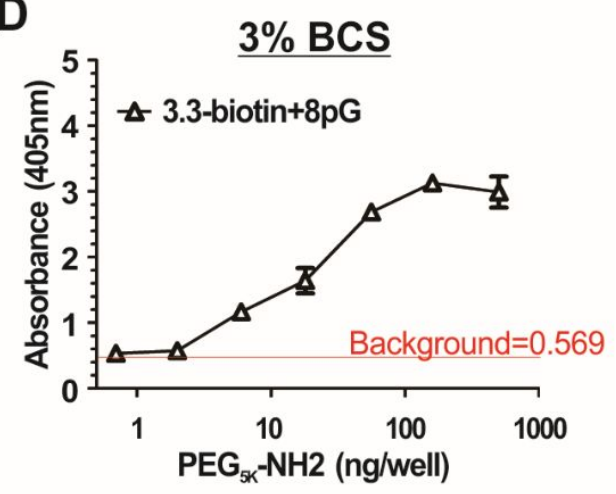

Figure S1. The interference of blocking reagents for the sensitivity and background value of 8pG based ELISAs. The microplates coated with different amount of $\mathrm{PEG}_{5 \mathrm{~K}}-\mathrm{NH}_{2}(0.69,2,6.2,18.5,55.5,166$ and $500 \mathrm{ng} / \mathrm{well}$ ) were blocked by (A)(C) 3\% (w/v) BSA and (B)(D) 3\% (w/v) BCS, respectively. Biotinconjugated anti-PEG antibody (termed 3.3-biotin, $1 \mu \mathrm{g} / \mathrm{mL}$ ) mixing with or without $8 \mathrm{pG}$ was added to these microplates, followed by the sequential addition of streptavidin-HRP and ABTS substrate. The representative data from three independent experiments are shown. The red line indicates the background value of each group. Bar, SD. 
A

B

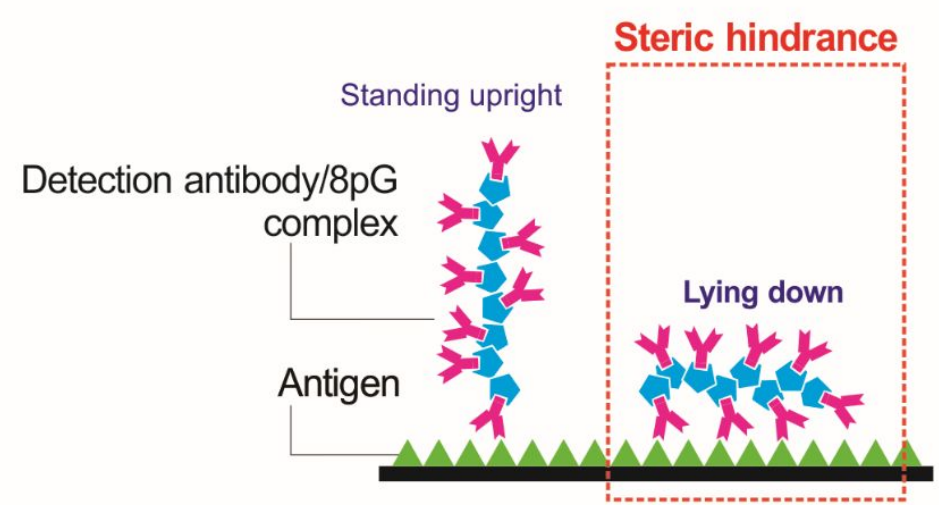

High antigen density

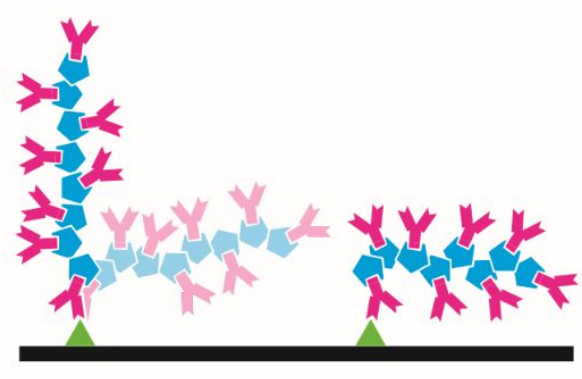

Low antigen density

Figure S2. Models of antibody/8pG complexes "standing upright" or "lying down" on (A) high density or (B) low density antigens. 
A

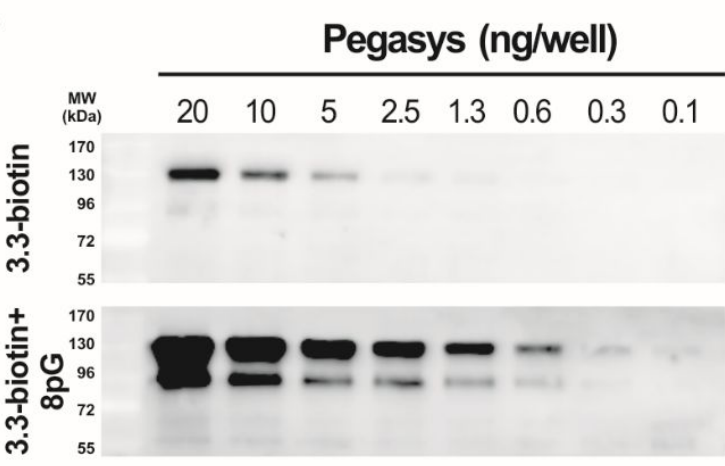

B

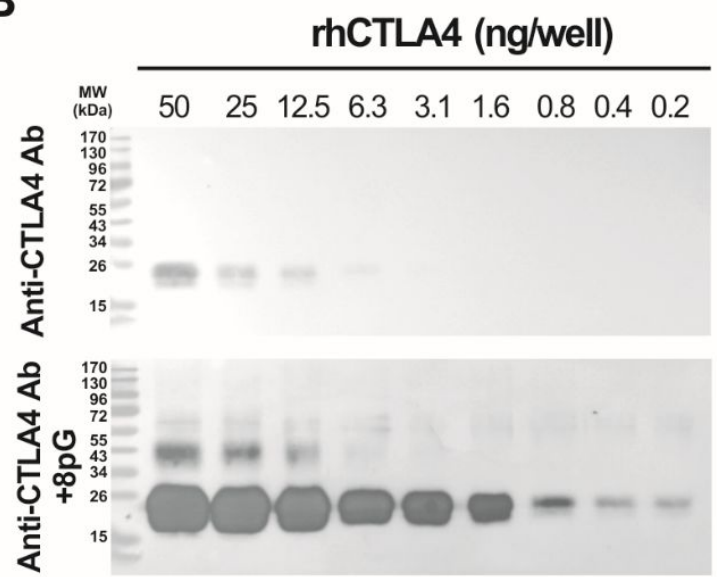

Figure S3. The raw images of Western blot data performed in the presence and absence of 8pG. Serial diluted (A) Pegasys and (B) rhCTLA4 were electrophoresed on a 10\% reducing SDS PAGE, transferred to nitrocellulose membrane, and probed with 3.3-biotin or anti-CTLA4 antibody which were mixed with or without $8 \mathrm{pG}$ as described in Materials and Methods section. 